\title{
Correlation between social factors and anxiety- depression in function dyspepsia: do relationships exist?
}

\author{
Zhen-Peng Huang ${ }^{1}$, Xian-Fen Yang ${ }^{2}$, Li-Sa Lan², Tao Liu², Cheng-Li Liu², Jin-Li Li², Zhong-Hui Liang ${ }^{2}$ \\ ${ }^{1}$ Department of Gastroenterology, Renmin Hospital of Wuhan University, Wuhan, Hubei, China \\ 2Department of Gastroenterology, 303 Hospital PLA China, Nanning, Guangxi, China
}

Prz Gastroenterol 2014; 9 (6): 348-353

DOI: $10.5114 /$ pg.2014.47897

Key words: digestion, function dyspepsia, anxiety, depression, socioeconomic factors.

Address for correspondence: Zhong-Hui Liang, Department of Gastroenterology, 303 Hospital PLA China, No. 52 Zhiwu Rd, Nanning 530022, Guangxi, China, phone: +8607712870095 , e-mail: 303xhlzh@163.com

\begin{abstract}
Introduction: Function dyspepsia (FD) may cause patients to suffer from anxiety and depression, and psychosocial disorders would have a significant effect on FD symptoms.

Aim: To examine the prevalence of anxiety and depression among function dyspepsia (FD) patients and to identify social factors of anxiety-depression among FD patients.

Material and methods: Patients with FD, who fulfilled the Rome III criteria, were enrolled. All patients were administered a validated Chinese version of the self-rating scale (SDS) and self-rating anxiety scale (SAS), and investigated regarding the patients' social factors.

Results: A total of 907 patients were enrolled, including 516 (56.89\%) FD patients within anxiety-depression status; SDS mean scores were $51.57 \pm 8.22$; SAS mean scores were $51.04 \pm 7.53 ; 52.28 \%$ were male and $64.25 \%$ were female $\left(\chi^{2}=262.54\right.$, $p<0.01$ ); $56.16 \%$ were aged $18-29$ years, $54.15 \%$ were aged $30-39$ years, $54.77 \%$ were aged $40-49$ years, $62.02 \%$ were aged $50-59$ years, $69.23 \%$ were aged above 60 years $\left(\chi^{2}=18.14, p<0.01\right) ; 67.44 \%$ were the retirees; $63.31 \%$ were manual workers; $55.10 \%$ were soldiers; $43.57 \%$ were mental workers; $38.89 \%$ were students $\left(\chi^{2}=716.53, p<0.01\right)$; $64.20 \%$ had junior high school degree or below; $57.36 \%$ had high school degrees; $42.03 \%$ had college degrees; $44.44 \%$ had master's or above degrees $\left(\chi^{2}=27.21, p<0.05\right) ; 38.10 \%$ were in good health condition; $61.90 \%$ were in poor health condition $\left(\chi^{2}=7.94, p<0.01\right) ; 20.31 \%$ had correlative family history; and $79.69 \%$ had no correlative family history $\left(\chi^{2}=2.23, p>0.05\right)$.

Conclusions: The FD patients have higher rates of anxiety and depression. Gender, age, occupation, education level, and health condition have a significant effect on anxiety and depression status. Female gender, advanced age, high-stress occupation, lower education level, and poor health condition all are risk factors. Family history has no relationship with anxiety and depression among FD patients.
\end{abstract}

\section{Introduction}

Function dyspepsia (FD) is highly prevalent all over the world [1, 2]. A previous study found that middle-aged and elderly people were prone to suffer from FD [3]. Although FD rarely affects patient mortality [4], it can have a bad impact on the quality of patients' lives [5]. However, there is a lack of available and effective treatment for the disease [6]. The symptoms may cause patients to suffer from anxiety and depression [7], then they may spend additional health care costs on their dyspepsia symptoms [8, 9], and they may have trouble in learning and working.
The mechanism of FD is not exactly clear, and it may be caused by motility disorders, visceral hypersensitivity, acid disorders, Helicobacter pylori infection, allergic disorders, psychosocial factors, and so on. Psychosocial disorders have a significant effect on FD [10]. Previous reports have confirmed that psychosocial stressors have affected FD symptoms [11]. Psychosocial disorders may result in disturbances in the modulation of the braingut axis, which may lead to FD symptoms [12, 13]. Psychosocial disorders can disturb patient's lives and make FD symptoms become worse, specially among FD overlap patients as well [14]. There are many factors that 
have an impact on anxiety and depression [15]. Finding anxiety and depression and their impact factors among FD patients [16-19].

\section{Aim}

This study is aimed at examining the prevalence of anxiety and depression among FD patients, and to identify social factors on anxiety and depression among FD patients.

\section{Material and methods}

The study was approved by the Institutional Ethical Committee of the $303^{\text {rd }}$ Hospital in PLA China. Informed consent was obtained from all participants before commencement of the study.

\section{Patient selection}

A total of 907 patients with FD, who fulfilled the Rome III criteria, were enrolled. There were 549 male and 358 female patients, aged $18-82$ years, mean 39.80 \pm 12.50 years. These were outpatients and inpatients who visited the department of gastroenterology in the $303^{\text {rd }}$ Hospital in PLA China from July 2012 to December 2013.

All patients underwent endoscopic examination, fulfilled the Rome III criteria, and were diagnosed with FD. According to the results of electrocardiogram, abdominal ultrasound, X-ray, blood test, all patients were ruled out for other diseases.

\section{Data collection}

Self-rating depression scale and self-rating anxiety scale

All patients were administered a validated Chinese version of Zung's self-rating depression scale (Self-Rating Scale, SDS) and self-rating anxiety scale (SAS) to identify patients' anxiety-depression status [20]. Specialized doctors used unified language to explain the scales to make the patients evaluate scales independently and without external factors of interference; for lower educational degree patients who could not evaluate scales independently, specialised doctors used reading scale items independently without suggestive language, and allowed patients to choose. Doctors calculated SAS and SDS standard scores, respectively. SAS and SDS standard scores $Y=$ int $(1.25 \mathrm{X})$. SDS standard score above 53 points was identified as depression, 53-62 points were catagorised as mild depression, 63-72 points were catagorised as moderate depression, higher than 72 points were catagorised as severe depression. SAS standard score above 50 points can be catagorised as anxiety, 50-59 points were catagorised as mild anxiety, 60-69 points were catagorised as moderate anxiety, and more than 70 points were identified as severe anxiety [21, 22].

\section{Investigation of social factors}

All patients were investigated regarding social factors of anxiety-depression, including gender, age, occupation (students, special occupations such as soldier, mental worker, manual worker, jobless, and retirees), educational degree (educational level below junior high school and in junior high school, high-school degree, college degree, master's degree and above), health condition, and family history [4].

\section{Statistical analysis}

All statistical analyses were assessed using SPSS for Windows, version 17.0. Continuous variables such as SDS and SAS scores are presented as mean \pm standard deviation (SD). Categorical variables such as gender, age, occupation, educational degree, family history, and living condition were expressed as proportions and percentages. Comparison of continuous variables was made using the $t$ test. Comparison of categorical variables was made using ANOVA and $\chi^{2}$ test. A two-sided $p$ value $<0.05$ was regarded as statistically significant.

\section{Results}

\section{Prevalence on anxiety and depression among function dyspepsia patients}

There were 516 (56.89\%) FD patients within anxiety and depression status, 73 (14.15\%) patients with depression status, 90 (17.44\%) patients within anxiety status, 353 (68.41\%) patients suffered from both anxiety and depression; 272 (63.85\%) patients had mild depression, 129 (30.28\%) patients had moderate depression, 25 (15.87\%) patients were in severe depression; 325 (73.37\%) patients had mild anxiety, 100 (22.57\%) patients had moderate anxiety, and 18 (4.06\%) patients had severe anxiety. Depression mean scores were $51.57 \pm 8.22$, and anxiety mean scores were $51.04 \pm 7.53$, among FD patients. In a comparison of SAS Chinese Normal People Norm Score $(n=1158$, norm score 29.78 \pm 0.46 ), mean anxiety scores were higher among the patients with FD $(t=1.965, p<0.05)$ (Figure 1$)$.

\section{Gender among function dyspepsia patients with anxiety-depression}

A total of 907 individuals of the FD participated in this study, 549 (60.53\%) were male and 358 (39.47\%) were female. Five hundred and sixteen (56.89\%) FD patients were within anxiety-depression status, 287 (52.28\%) were male, and 230 were (64.25\%) female $\left(64.25 \%\right.$ vs. $\left.52.28 \%, \chi^{2}=262.54, p<0.01\right)$. 
Female FD patients were much more likely to suffer from anxiety and depression than were males.

\section{Age among function dyspepsia patients with anxiety-depression}

From a total of 907 patients with FD, 219 (24.15\%) patients were aged $18-29$ years, 253 (27.89\%) patients

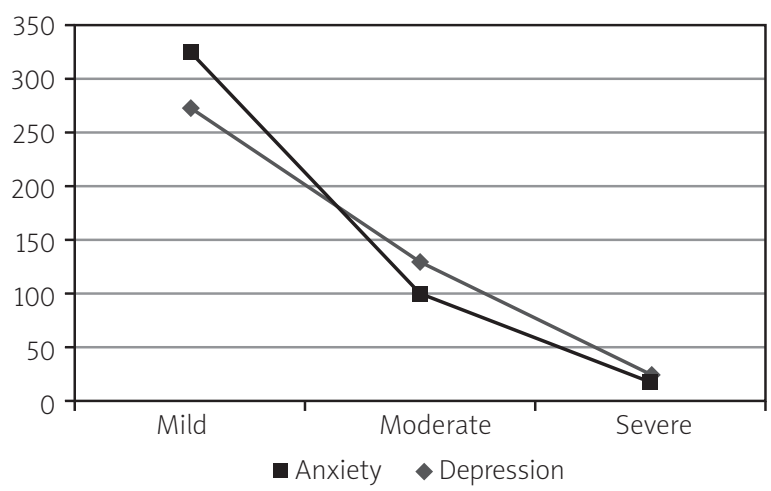

Figure 1. Prevalence on anxiety and depression among FD patients

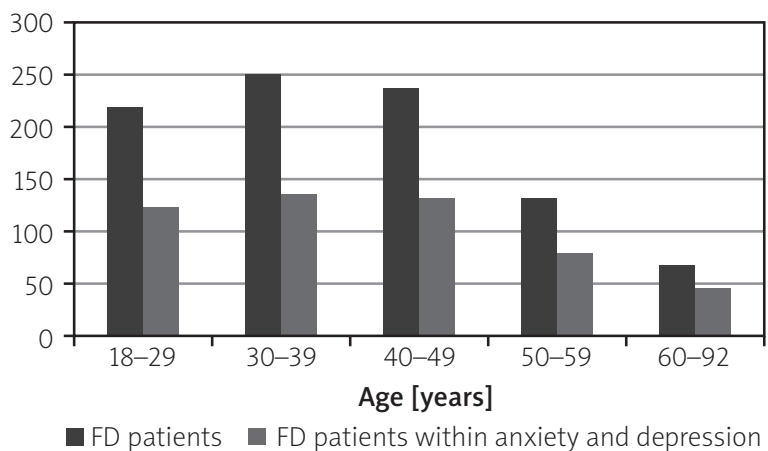

Figure 2. Correlation between age and anxietydepression among FD patients

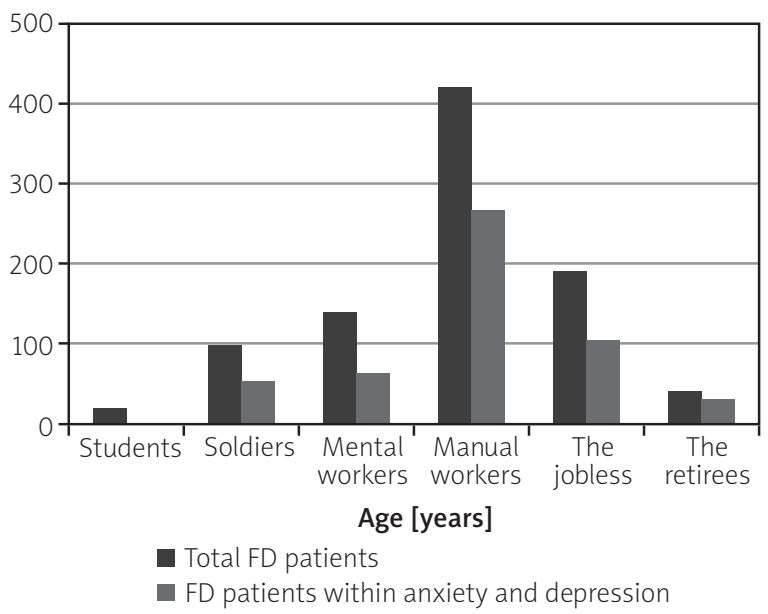

Figure 3. Correlation between occupation and anxiety-depression among FD patients were aged 30-39 years, 241 (26.57\%) patients were aged $40-49$ years, 129 (14.22\%) patients were aged 50-59 years, and $65(7.17 \%)$ patients were aged above 60 years old. For FD patients with anxiety and depression, 123 (56.16\%) were aged 18-29 years, 137 (54.15\%) were aged 30-39 years, 132 (54.77\%) were aged 40 -49 years, $80(62.02 \%)$ were aged $50-59$ years, and $45(69.23 \%)$ were aged above 60 years.

The aged FD patients were much more likely to suffer from anxiety and depression than the young patients $\left(\chi^{2}=18.14, p<0.01\right)$ (Figure 2$)$.

\section{Occupation among function dyspepsia patients with anxiety and depression}

From a total of 907 patients with FD, 417 (45.98\%) were manual workers, including factory employees farmers, drivers, and so on. One hundred and ninety-one (21.06\%) were jobless, 140 (15.44\%) were mental workers, including teachers, businessmen, doctors, office workers, and so on. Ninety-eight (10.80\%) were soldiers, 43 (4.74\%) were retirees, and 18 (1.98\%) were students. For FD patients with anxiety-depression, 29 (67.44\%) were retirees, 264 (63.31\%) were manual workers, $54(55.10 \%)$ were soldiers, $61(43.57 \%)$ were mental workers, and (38.89\%) were students.

The FD patients who were retired, out of work, or had low-wage jobs, such as manual workers or unemployed, or in high-stress occupations such as soldiers, were more likely to suffer from anxiety-depression $\left(\chi^{2}=716.53, p<0.01\right)$ (Figure 3).

\section{Education level among function}

dyspepsia patients with anxiety-depression

From a total of 907 patients with FD, 433 (47.74\%) patients had educational level below junior high school and on junior high school degree, 258 (28.45\%) had a high school degree, 207 (22.82\%) had a college degree, and 9 (0.99\%) had a master's degree or above. Within anxiety-depression patients, 278 (64.20\%) were below junior high school and on junior high school degree level, 148 (57.36\%) were at high school degree level, 87 (42.03\%) had a college degree, and 4 (44.44\%) had a master's degree and above.

Lower education degree can increase the risk for FD patients suffering from anxiety-depression $\left(\chi^{2}=27.21\right.$, $p<0.05$ ) (Figure 4).

\section{Health care condition among function dyspepsia patients within anxiety- depression}

From a total of 907 patients with FD, 525 (57.88\%) had poor health care level, and 382 (42.12\%) patients' 
health care condition was good. For FD patients with anxiety-depression, 320 (61.90\%) had poor health care condition, and 197 (38.10\%) had good health care condition.

Poor health care condition can increase the risk for FD patients with anxiety-depression $(61.90 \%$ vs. $38.10 \%$, $\left.\chi^{2}=7.94, p<0.01\right)$

\section{Family history among function dyspepsia patients with anxiety-depression}

From a total of 907 patients with FD, 738 (81.37\%) had no correlative family history, and 169 (18.63\%) had correlative family history. Among anxiety-depression patients, 412 (79.69\%) had no correlative family history, and 105 (20.31\%) had correlative family history.

However, there is no statistically significant impact of family history on the FD patients regarding their suffering from anxiety-depression or not (79.69\% vs. $20.31 \%, \chi^{2}=2.23, p>0.05$ ).

\section{Discussion}

The results of present study confirmed that anxiety and depression of the patients suffered from mild anxiety and depression, which was also found in previous research [23]. The present study found that all FD patients had significantly higher mean scores for anxiety and depression than Chinese Normal People Norm Score, and the prevalence of anxiety and depression were also higher among FD patients. The prevalence of anxiety and depression is reflective of FD patients who were more likely to consult for their digestive symptoms. Anxiety is possibly one of the important reasons for FD patients to ask for medical consultation. Studies of Chinese and Western patients draw similar conclusions $[24,25]$.

The present study showed that gender and age had an effect on the FD patients with anxiety and depression. Female and aged FD patients had a significantly higher prevalence of anxiety and depression than FD patients without anxiety and depression. The latest report confirmed that the prevalence in females, specially among postmenopausal women with FD, is higher than in men $[26,27]$. The older FD patients would take excessive care of their own state of health, and FD symptoms would easily to make them suffer from anxiety and depression [28].

This study supported the theory that socioeconomic status has a significant impact on anxiety and depression in FD patients. The present study showed that patients with lower socioeconomic status such as low-wage workers or jobless, lower educational degree, poor health care condition, and work in high-stress occupations such as soldiers and mental workers, had

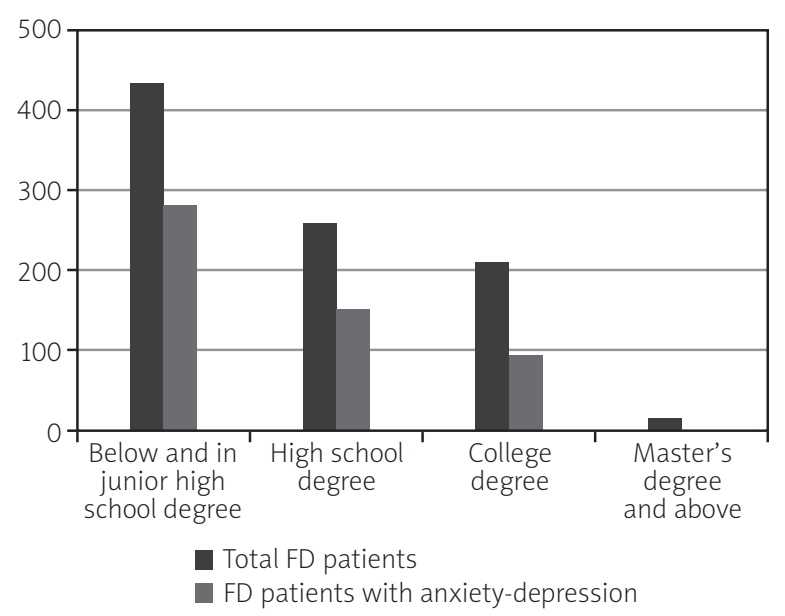

Figure 4. Correlation between education degree and anxiety-depression among FD patients

a significantly higher prevalence of anxiety and depression than other FD patients. A previous report pointed out that chronic social stressors were highly prevalent in FD patients with anxiety and depression [29, 30]. The latest studies show that major socioeconomic and healthcare conditions are highly prevalent for FD, and chronic stress was one of the prominent features of FD [31, 32]. Patients who had lower socioeconomic status or worked in high-stress occupations suffer from much more stress, and poor health care condition did not ensure that FD patients who had lower socioeconomic status consulted a doctor when necessary.

In contrast, in this study we found that there was no relationship between correlative family history and FD patients with anxiety and depression. A survey among Thai schoolchildren found that there was no significant difference in family history and dyspepsia morbidity [33].

A program of psychological support, including behavioural activation, psychological education, relaxation training, interpersonal training, and so on, among FD patients with anxiety and depression would improve FD patients' psychological disorders. A previous metaanalysis showed that behavioural activation was an effective treatment for patients with depression [34]. A prospective randomised trial found that psychoeducational information by video could help reduce anxiety in patients [35].

Several limitations of the study should be highlighted. As this study was hospital-based, the findings are not generalisable to all FD patients who are unable to consult a doctor. Patients all came from Asia and so are not be generalisable to the FD patients in other countries.

\section{Conclusions}

This study has confirmed that FD patients have higher rates of anxiety and depression, and most pa- 
tients have suffered from both mild anxiety and mild depression. Gender, age, and socioeconomic status such as occupation, education degree, and health care condition, have significant effects on anxiety and depression status. Female gender, advanced age, high-stress occupations, lower educational degree, and poor health care conditions all are risk factors. Family history has no relationship with anxiety and depression among FD patients.

\section{Acknowledgments}

We should like to thank the staff of the Department of Gastroenterology in the $303^{\text {rd }}$ Hospital PLA China for their assistance in this study.

\section{References}

1. Mahadeva S, Goh KL. Epidemiology of functional dyspepsia: a global perspective. World J Gastroenterol 2006; 12: 2661-6.

2. Egloff N, Beer C, Gschossmann JM, Sendensky AH, von Känel R. Pathogenesis of functional gastrointestinal disorders - an interdisciplinary Perspective. Praxis (Bern 1994) 2010; 99: 419-27.

3. Piotrowicz G, Stępień B, Rydzewska G. Socio-demographic characteristics of patients with diagnosed functional dyspepsia. Prz Gastroenterol 2013; 8: 354-65.

4. Hartono JL, Mahadeva S, Goh KL. Anxiety and depression in various functional gastrointestinal disorders: do differences exist? J Digest Dis 2012; 13: 252-7.

5. Lee SW, Lee TY, Lien HC, et al. The risk factors and quality of life in patients with overlapping functional dyspepsia or peptic ulcer disease with gastroesophageal reflux disease. Gut Liver 2014; 8: 160-4.

6. Lee H, Jung HK, Huh KC. Current status of functional dyspepsia in Korea. Korean J Intern Med 2014; 29: 156-65.

7. Wilhelmsen I. Somatization, Sensitization, and functional dyspepsia. Scand J Psychol 2002; 43: 177-80.

8. Mapel DW. Functional disorders of the gastrointestinal tract: cost effectiveness review. Best Pract Res Clin Gastroenterol 2013; 27: 913-31.

9. Lacy BE, Weiser KT, Kennedy AT, et al. Functional dyspepsia: the economic impact to patients. Aliment Pharmacol Ther 2013; 38: 170-7.

10. Voiosu TA, Giurcan R, Voiosu AM, Voiosu MR. Functional dyspepsia today. Maedica (Buchar) 2013; 8: 68-74.

11. Budavari Al, Olden KW. Psychosocial aspects of functional gastrointestinal disorders. Gastroenterol Clin North Am 2003; 32: 477-506

12. Mayer EA, Tillisch K, Bradesi S. Review article: modulation of the brain-gut axis as a therapeutic approach in gastrointestinal disease. Aliment Pharmacol Ther 2006; 24: 919-33.

13. Mayer EA, Tillisch K. The brain-gut axis in abdominal pain syndromes. Annu Rev Med 2011; 62: 381-96.

14. Lee HJ, Lee SY, Kim JH, et al. Depressive mood and quality of life in functional gastrointestinal disorders: differences between functional dyspepsia, irritable bowel syndrome and overlap syndrome. Gen Hosp Psychiatry 2010; 32: 499-502.

15. Hojo M, Miwa H, Yokoyama T, et al. Treatment of functional dyspepsia with antianxiety or antidepressive agents: systematic review. J Gastroenterol 2005; 40: 1036-42.
16. Seno H, Nakase H, Chiba T. Usefulness of famotidine in functional dyspepsia patient treatment: comparison among prokinetic, acid suppression and antianxiety therapies. Aliment Pharmacol Ther 2005; 21 Suppl. 2: 32-6.

17. Moshiree B, Barboza J, Talley N. An update on current pharmacotherapy options for dyspepsia. Expert Opin Pharmacother 2013; 14: 1737-53.

18. Lacy BE, Talley NJ, Locke GR 3rd, et al. Review article: current treatment options and management of functional dyspepsia. Aliment Pharmacol Ther 2012; 36: 3-15.

19. Talley NJ. Functional gastrointestinal disorders as a public health problem. Neurogastroenterol Motil 2008; 20 Suppl. 1: 121-9.

20. Xiangdong $W$, Xilin W, Hong $M$, et al. The mental health scale revised. Chin Mental Health J 1999; 12: 194-6, 235-8.

21. Zung WW. Depression Status Inventory and Self-Rating Depression Scale. ECDEU Assesssment Manual for Psychoparmacology. Revised 1976. William Guy. U.S. Department of Health Education and Welfare Public Health Service p. 172-8.

22. Zung WWK. A rating instrument for Anxiety disorders. Psychosomatics 1971; 12; 371-9.

23. Aro P, Talley NJ, Ronkainen J, et al. Anxiety is associated with uninvestigated and functional dyspepsia (Rome III criteria) in a Swedish population-based study. Gastroenterology 2009; 137: 94-100.

24. Hu WH, Wong WH, Lam CL, et al. Anxiety but not depression determines health care-seeking behaviour in Chinese patients with dyspepsia and irritable bowel syndrome: a population-based study. Aliment Pharmacol Ther 2002; 30: 643-51.

25. Koloski NA, Talley NJ, Boyce PM. Predictors of health care seeking for irritable bowel syndrome and nonulcer dyspepsia: a critical review of the literature on symptom and psychosocial factors. Am J Gastroenterol 2001; 96: 1340-9.

26. Lee SW, Lee TY, Lien HC, et al. The risk factors and quality of life in patients with overlapping functional. Gut Liver 2014; 8: 160-4.

27. Cain KC, Jarrett ME, Burr RL, et al. Gender differences in gastrointestinal, psychological, and somatic symptoms in irritable bowel syndrome. Dig Dis Sci 2009; 54: 1542-9.

28. Li R, Zhu X, Yin S, et al. Multimodal intervention in older adults improves resting-state functional connectivity between the medial prefrontal cortex and medial temporal lobe. Front Aging Neurosci 2014; 10: 39.

29. Craig TK, Brown GW. Goal frustration and life events in the etiology of painful gastrointestinal disorder. J Psychosom Res 1984; 28: 411-21.

30. Bennett E, Beaurepaire J, Langeluddecke P, et al. Life stress and non ulcer dyspepsia: a case-control study. J Psychosom Res1991; 35: 579-90.

31. Altan E, Masaoka T, Farré R, Tack J. Acotiamide. A novel gastroprokinetic for the treatment of patients with functional dyspepsia: postprandial distress syndrome. Expert Rev Gastroenterol Hepatol 2012; 6: 533-44.

32. Bennett EJ, Piesse C, Palmer K, et al. Functional gastrointestinal disorders: psychological, social, and somatic features. Gut 1998; 42: 414-20.

33. Phavichitr N, Koosiriwichian K, Tantibhaedhyangkul R. Prevalence and risk factors of dyspepsia in Thai schoolchildren. J Med Assoc Thai 2012; 95 Suppl. 5: S42-7. 
34. Ekers D, Webster L, Van Straten A, et al. Behavioural activation for depression; an update of meta-analysis of effectiveness and sub group analysis. PLoS One 2014; 9: e100100.

35. Arabul M, Kandemir A, Celik M, et al. Impact of video information before unsedated upper gastrointestinal endoscopy on patient satisfaction and anxiety: a prospective randomized trial. Prz Gastroenterol 2013; 8: 44-9.

Received: 22.04.2014

Accepted: 6.07.2014

\section{Annex}

Annex 1. Self-rating depression scale (SDS)

For each item below, please check the column which best describes how often you felt or behaved this way during the past several days. All columns could divide into "a little of the time", "some of the time", "a good part of the time", "most of the time".

1. I feel down hearted and blue.

2. Morning is when I feel the best.

3. I have crying spells or feel like it.

4. I have trouble sleeping at night.

5. I eat as much as I used to.

6. I still enjoy sex.

7. I notice that I am losing weight.

8. I have trouble with constipation.

9. My heart beats faster than usual.

10. I get tired for no reason.

11. My mind is as clear as it used to be.

12. I find it easy to do the things I used to.

13. I am restless and can't keep still.

14. I feel hopeful about the future.

15. I am more irritable than usual.

16. I find it easy to make decisions.

17. I feel that I am useful and needed.

18. My life is pretty full.

19. I feel that others would be better off if I were dead.

20. I still enjoy the things I used to do.

\section{Annex 2. Self-rating anxiety scale (SAS)}

For each item below, please check the column which best describes how often you felt or behaved this way during the past several days. All columns could divide into "a little of the time", "some of the time", "good part of the time", "most of the time".

1. I feel more nervous and anxious than usual.

2. I feel afraid for no reason at all.

3. I get upset easily or feel panicky.

4. I feel like I'm falling apart and going to pieces.

5. I feel that everything is all right and nothing bad will happen.

6. My arms and legs shake and tremble.

7. I am bothered by headaches, neck and back pain.

8. I feel weak and get tired easily.
9. I feel calm and can sit still easily.

10. I can feel my heart beating fast.

11. I am bothered by dizzy spells.

12. I have fainting spells or feel like it.

13. I can breathe in and out easily.

14. I get numbness and tingling in my fingers and toes.

15. I am bothered by stomach aches or indigestion.

16. I have to empty my bladder often.

17. My hands are usually dry and warm.

18. My face gets hot and blushes.

19. I fall asleep easily and get a good night's rest.

20. I have nightmares. 